Research Paper

\title{
Quantification of Blood-Brain-Barrier Permeability Dysregulation and Inflammatory Activity in MS Lesions by Dynamic-Contrast Enhanced MR Imaging
}

\author{
Mohammad Ali Oghabian ${ }^{1^{*}}$ (D), Asieh Fatemidokht ${ }^{2}$ (D), Mohammad Hossein Haririchian ${ }^{3}$ (D)
}

1. Department of Neuroimaging and Analysis, Research Center for Cellular and Molecular Imaging, Tehran University of Medical Sciences, Tehran, Iran 2. Department of Biomedical Engineering and Medical Physics, Faculty of Medicine, Tehran University of Medical Sciences, Tehran, Iran.

3. Iranian Center of Neurological Research, Neuroscience Institute, Imam Khomeini Hospital Complex, Tehran University of Medical Sciences, Tehran, Iran

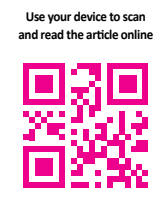

ditation Oghabian, M. A., Fatemidokht, A., Haririchian, M. H. (2022). Quantification of Blood-Brain-Barrier Permeability Dysregulation and Inflammatory Activity in MS Lesions by Dynamic-Contrast Enhanced MR Imaging. Basic and Clinical Neuroscience, 13(1), 117-128. http://dx.doi.org/10.32598/bcn.2022.575.1

doi) $h$ http://dx.doi.org/10.32598/ben.2022.575.1

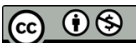

Article info:

Received: 01 Jun 2019

First Revision: 08 Aug 2020

Accepted: 09 Sep 2020

Available Online: 01 Jan 2022

Keywords:

Blood-Brain-Barrier, Inflammatory activation, Multiple sclerosis

\section{A B S T RA C T}

Introduction: blood-brain-barrier perfusion characterization impaired in MS as some studies have shown recently but a comparison between perfusion parameters in contrast-enhanced and non-enhanced lesions not have been well documented. Pharmacokinetic quantitative parameters have obtained from dynamic contrast-enhanced in magnetic resonance imaging is a useful way to quantify blood-brain barrier permeability leakage.

Methods: MR examination was performed on 28 patients with Relapsing-remitted Multiple Sclerosis (RRMS) with (Mean \pm SD age: $34.7 \pm 9.28)$ which had multiple lesions in the brain.3D dynamic T1-weighted spoiled gradient echo was obtained and Perfusion parameters and its map assessed in enhanced and non-enhanced lesions after intravascular injection differences in parameters and map obtained by analyzing ROI in Extended Toft model.

Results: permeability as measured Krtans was a significantly higher value in CE to compare $\mathrm{NE}$ lesions. Ktrans and Kep have significant differences in NAWM and CE and NE lesions. Vb was slightly different in NE and CE lesions.

Conclusion: Permeability measured as Ktrans was the good parameter to show permeability impairment of BBB in CE lesions. Dysregulation in BBB is an acceptable sign to indicate existence inflammation in CE lesions.

\section{* Corresponding Author:

Address: Department of Neuroimaging and Analysis, Research Center for Cellular and Molecular Imaging, Tehran University of Medical Sciences, Tehran, Iran.

Tel: +98 (21) 66907519

E-mail: oghabian@sian.tums.ac.ir 


\section{Highlights}

- Multiple Sclerosis,

- Inflammation,

- Blood-brain-barrier dysregulation

\section{Plain Language Summary}

Inflammation activity in MS patients has an important role to cause BBB dysfunction.in this article to achieve results to confirm the inflammation importance in MS patients with acute lesions. MRI modality have been used and with comparison between acute and chronic lesions and NAWM of MS patient's presence of inflammation have been proved.

\section{Introduction}

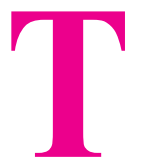

he Blood-Brain Barrier (BBB) is a complex structure of cerebral endothelial cells and pericytes, enclosed and supported by brain resident immune cells like astroglia. In the Multiple Sclerosis (MS) brain, activated peripheral lymphocytes are infiltrated in the central nervous system to begin an immune response that damages myelin and axons. Pro-inflammatory cytokines and reactive oxygen and nitrogen species are accumulated there and may lead to myelin damage. Abnormality in the BBB function and endothelial movement of activated leukocytes from the earliest cerebrovascular breakdown is due to the release of inflammatory cytokines (Ortiz et al., 2014; Varatharaj \& Galea, 2017).

Assessment of BBB permeability dysfunction is a therapeutic marker and hallmark of subtle changes in lesions and Normal-Appearing White Matter (NAWM) in MS (Cramer, Simonsen, Frederiksen, Rostrup, \& Larsson, 2014; Gaitan et al., 2011). The early pathological findings in MS are in vivo detection of subtle radiological changes in NAWM. Quantitative and semi-quantitative MRI studies support the relationship between inflammation and higher permeability in BBB, as evidenced by the high distribution of contrast agents in MS-enhanced acute lesions compared with non-enhanced chronic lesions. MRI-contrast agent Gd (Gadolinium), based on its unique physicochemical properties, could detect acute inflammatory lesions.

Also, to display contrast agent abilities better, the acquisition protocol's properties like the delay between administrating CA (Contrast Agent) and acquisition parameters have essential roles. It is to be noted that Gd-CA could not display all types of inflammation, such as glial cell activation, which contributes to dysfunction (Absinta, Sati, \& Reich, 2016; Tourdias \& Dousset, 2013). Acute active plaques are usually observed in relapsingremitting MS and are shown as the pathologic reason for attacks in acute MS patients. Acute active MS lesions are demyelinated plaques surrounded by macrophages which could be the result of a fracture in the BBB due to inflammation and high activation of brain immune cells. Besides, the axonal loss is an essential event in old chronic plaques that MRI-CA cannot be detected by any infiltration of inflammatory factors in these lesions. However, these lesions could be detected by perfusion imaging. So perfusion parameters can show alteration in $\mathrm{BBB}$ and the presence of inflammation in different types of MS lesions (Popescu, Pirko, \& Lucchinetti, 2013).

To investigate the BBB function and its alteration, SPECT (single-photon emission computerized tomography) is an imaging method used in stroke (Nagaraja, Nagesh, Ewing, Whitton, Fenstermacher, \& Knight 2007; Gilad, Lampl, Eilam, Boaz, \& Loyberboim, 2012). Also, some modalities like dynamic susceptibility contrast and dynamic contrast-enhanced of MRI are currently used to quantify variation in blood flow and volume in different pathology in the brain (Roberts, Roberts, Brasch, \& Dillon, 2000; Li et al., 2014; Filippi \& Rocca, 2011). Dynamic contrast susceptibility was more used to study hemodynamic changes like cerebral blood flow and cerebral blood volume. So, many studies used this method to quantify perfusion parameters in MS lesions and have been performed recent developments in analysis methods to reach an accurate and unbiased value of perfusion alteration in lesions and NAWM (Sowa et al., 2015; Ge et al., 2005). Also, some studies have shown BBB dysfunction in gray matter and white matter of relapsing-remitting MS patients when measured by MRI techniques like 
Dynamic-Susceptibility Contrast (DSC) MRI. This technique could reveal inflammation not detectable in routine T1-weighted MRI (Eftekhari et al., 2017).

It has been shown that Dynamic Contrast-Enhanced (DCE)-MRI can combine perfusion and permeability measurements; it was introduced recently as an appropriate modality to study subtle changes in BBB (Armitage, Farrall, Carpenter, Doubal, \& Wardlaw, 2011; Bae et al., 2018). So perfusion-weighted modality techniques could detect local and diffuse low-grade inflammatory variations not shown in routine MRI. MRI-perfusion modality could measure the integrity of the BBB structure by using Gd-CA as an in-vivo marker to show lowgrade neuroinflammation. The hyperintense signal in T1-weighted MRI images has been observed because of high permeability in the BBB due to neuroinflammation presence which has been enabled the extravasation of Gd-CA among tight endothelial cells of BBB (Hagens, van Berckel, \& Barkhof, 2016). Also, many researchers assessed quantitative perfusion parameters to identify leakage in BBB in MS brains by obtaining values of plasma flow constant (Ingrisch et al., 2012; Cramer \& Larsson, 2014; Taheri, Rosenberg, \& Ford, 2013).

To analyze data in DCE-MRI, different models are available to estimate kinetic parameters. Most analyzing methods of this modality have analyzed the compartment model to measure some composition of the three primary parameters: the transfer constant (Ktrans), the Extravascular Extracellular Space (EES) fractional volume $(\mathrm{Vb})$, and the rate constant (Kep). Toft began the DCE-MRI method analyzing research. His models supplied a complete description to use compartment-based models to measure approximately physiologically reasonable parameters from a ROI (Region of Interest) and identified these models based on the relationship between the three mentioned parameters. So analyzing models comprise standard Toft-Kety, extended Toft, Patlak, and two compartmental models. The standard and modified Toft models assume the blood plasma and EES as two compartments. Both models consider a wellmixed tracer in each compartment in a similar concentration. The two models could supply data due to the distribution of contrast agents in the two spaces (Tofts et al., 1999; Schabel, 2012).

In MS, gadolinium enhancement could prepare a direct display of breaches in the BBB of in brain that accompany active lesions, and so it is used to detect the number of new plaques in the CNS (usually the brain) of MS. It, therefore, provides a good measure of disease activity and helps distinguish between acute (or active) plaques and chronic (or non-active) lesions (Barkhof, 2002); administration of a Gd-CA has been used in the routine MR imaging protocol in MS, and it is helpful to detect acute MS lesions based on a local infraction in the BBB due to acute neuroinflammation. Mostly, it would be desirable to improve a quantitative method to discriminate between enhanced lesions with inflammation and nonenhanced lesion with degenerated or tissue scar. Previous studies have shown alterations in active MS lesions based on MR imaging frequency shift (Wiggermann et al., 2013), magnetization transfer ratio (Levesque et al., 2010), and relaxation values (Jurcoane et al., 2013). These techniques suggest the possibility of quantitative methods' abilities to distinguish and characterize the active lesions by other modalities of MRI.

This work aims to provide measurements of quantitative perfusion MRI in Contrast-Enhanced (CE), NonContrast-Enhanced (NCE) lesions, and NAWM in MS brains to compare the value of perfusion parameters, like Ktrans, Kep, and Vb by extended Toft analyzing model to show impairment in BBB and existence of inflammatory activity in $\mathrm{CE}$ plaques.

\section{Methods}

\section{Study subjects}

A total of 28 RRMS patients based on MacDonald Criteria (Thompson et al., 2018) were accepted in the MS Clinic at the Research Institute of Neurology at the Imam Hospital in Tehran City, Iran. All patients signed written consent, and the study was approved by the Ethics Committee of Tehran University of Medical Sciences. Patients were examined by 3-T MRI and were classified as patients with enhanced lesions and non-enhanced lesions. Table 1 presents the demographic properties of patients in detail. The RRMS patients have a Mean \pm SD disease duration of about $10 \pm 2.5$ years, and the Expanded Disability Status Scale (EDSS) range of patients was changed between 0 and 4, scaled by a resident neurologist in the MS clinic. The most used drug in patients of two groups was interferon beta-1a (like CinnoVex and ReciGen), with a maximum biological response of about $48 \mathrm{~h}$. So the images were obtained about 2 days after administration of the last dose of the consumed drugs. MR images were obtained based on a routine MS protocol, and multi-parametric MRI sequences were added before and after the administration of a contrast agent. The images were obtained at most 7 days after the attack in patients diagnosed as a relapse by the neurologists. The last prescription of corticosteroids in RRMS (Relapsing-remitted Multiple Sclerosis) patients was at least 
2 months before the attack because anti-inflammation drugs influence the activity of lesions.

\section{MRI imaging protocols}

Images were obtained on a 3T MR imaging scanner (GE: GE Healthcare, Chicago, Illinois, United States) using a 24-channel phased-array head coil. The sequence parameters for routine protocol and PWI images were as follows:

- 2D-T2 weighted FLAIR: 23 images acquisition; voxel size, $0.45 \times 0.45 \times 5.5 \mathrm{~mm}$; TE, $120 \mathrm{~ms}$; TR, $8000 \mathrm{~ms}$; TI, $2350 \mathrm{~ms}$; scan time, 3.05 minutes;

- 2D T1-weighted SE pre and post-Gd injection: 23 images acquisition; voxel size, $0.45 \times 0.45 \times 5.5 \mathrm{~mm}$; TE, $11 \mathrm{~ms}$; TR, $600 \mathrm{~ms}$; scan time, 3:08 min;

- DCE-MRI: 3D-SPGR: 1080 images acquisition; voxel size, $2 \mathrm{~mm}$; TE, $1.29 \mathrm{~ms}$; TR, $3.34 \mathrm{~ms}$; TI, 5ms; saturation FA, 12 degrees; scan time, 4:09 min.

For all patients, a single dose $(0.2 \mathrm{~mL} / \mathrm{kg})$ of gadolinium (DOTAREM; Guerbet, Aulnay, France) was used at an injection rate of $3 \mathrm{~mL} / \mathrm{min}$, followed by a saline chase of $20 \mathrm{~mL}$.

\section{Image analysis}

Regions of Interest (ROIs) were drawn semi-manually by an expert radiologist. First, in post-contrast-T1, ROI was located on enhanced active lesions. Then, ROIs were masked and located on T2-FLAIR images in MRIcro (Nottingham, UK). In chronic lesions without any enhancement, ROIs were placed on selected lesions in T2-FLAIR images. Finally, ROIs were located on obtained images by perfusion sequence. Then, ROIs were analyzed, and a map of perfusion parameters was obtained using the DCE Tools plugin (version 2.0SP1) within ClearCanvas (Toronto, Ontario, Canada) framework (Figure 1). The DCE Tools allow selecting contrast uptake models in each ROI and this study. The modified Toft model has been chosen (Tofts et al., 1999). The used analytical model describes two compartments of EES and blood plasma and measures a map of three kinetic tracer parameters in contrast uptake curves. Trans, Kep, and $\mathrm{Vb}$ are three important perfusion parameters that have been computed by DCE Tools. Among these parameters, Ktrans showed the amount of permeability of the BBB and played an important role in demonstrating its dysfunction. The Arterial Input Function (AIF) was manually placed in the middle cerebral artery into the two-compartment model. Here, AIF corrects the kinetic analysis by measuring the signal from an ROI in MCA on T1-weighted images and setting the results.

\section{Statistical analysis}

All data were analyzed in SPSS software v. 21 (SPSS Inc., Chicago, IL, USA). To investigate perfusion parameters difference between enhanced and non-enhanced lesions, the Mann-Whitney test was used because of the nonlinearity in parameters. To compare statistical values between non-enhanced lesions and enhanced-lesions and NAWM separately, the Wilcoxon Ranked test was used.

\section{Results}

A total of 26 lesions in 13 men and 13 women with RRMS (based on MacDonald criteria) were investigated. All lesions were located in the periventricular, juxtacortical, and temporal lobes. The number of male and female patients was equal to eliminate sex effects because this disease was observed in women. Lesions were categorized based on taking uptake contrast agents into enhanced and non-enhanced. In each patient, measurements were obtained in lesions and NAWM to compare parameters pairwise. Perfusion parameters were measured and analyzed in all chronic and active lesions. Figure 1 (parts $\mathrm{A}, \mathrm{B}$, and C) shows the ROI drawn on the original image from one patient with an active lesion. The pharmacokinetic parameters map in active lesions is indicated in Figure 1 (parts D, E, and F). Values of permeability parameters (Ktrans, Vb, Kep) are presented in Table 2, indicating a considerable difference between these parameters between CE lesions and NAWM and NCE lesions (Figure 2), measured by the modified Toft model.

As seen in Table 3, the mean value of Ktrans in enhanced lesion had a significant difference $(\mathrm{P}<0.001)$ with the mean value of non-enhanced lesions; Ktrans values also indicated a significant difference $(\mathrm{P}<0.05)$ between enhanced lesion and NAWM. However, there was no considerable difference between non-enhanced lesions and NAWM (Figure 3). Also, Vb mean value, as seen in Table 4, shows that the difference between means in enhanced and non-enhanced lesions was slightly significant $(\mathrm{P}<0.057)$. However, $\mathrm{Vb}$ did not significantly differ $(\mathrm{P}=0.06)$ between the Mean $\pm \mathrm{SD}$ of $\mathrm{CE}$ lesions and NAWM. Also, NCE lesion's mean and NAWM mean $0.15 \pm 0.08$ lacked a significant difference (Figure 4). Kep value in Table 4 also shows a significant difference $(\mathrm{P}<0.001)$ between the mean of CE lesions and NAWM. However, NCE lesions mean and NAWM had significant differences $(\mathrm{P}<0.05)$, but this parameter in $\mathrm{CE}$ le- 
Table 1. Patient's demographic information

\begin{tabular}{cc}
\hline Subjects & $\mathbf{2 6}$ \\
\hline Median, the standard deviation of age & 34.789 .28 \\
\hline Sex(M/F) & 0.75 \\
\hline No of a patient with enhanced lesions & 13 \\
\hline No of patients with non-enhanced lesions & 13 \\
\hline The range of EDSS & $0-4$ \\
\hline Duration of disease(yrs) & $10 \pm 2.5$ \\
\hline Last replace (days) & $1-7$ \\
\hline Last prescription Corticosteroids (months) & 2 \\
\hline
\end{tabular}

sions mean, and NCE lesions mean lacked any significant difference $(\mathrm{P}=0.18)$ (Figure 5).

\section{Discussion}

In this study, we measured perfusion parameters (Ktrans, Kep, and $\mathrm{Vb}$ ) in acute lesions with Gd contrast agent uptake and chronic lesions without enhancement after injection of Gd and their NAWM with using T1DCE MRI and modified Toft analyzing method. We wanted to investigate BBB permeability impairment and inflammatory activation in MS patients. Our results showed a significant difference in Ktrans and Kep of CE lesions compared to NCE lesions and NAWM, as observed in Figure 2. The results confirm that Ktrans as a biomarker can distinguish between acute and chronic lesions. Also, Kep has a higher value in CE lesions compared to NAWM. Vb results lack any significant difference between CE, NCE lesions, and NAWM. Previous studies showed abnormalities in BBB in White Matter (WM) and MS lesions in the different regions of the brain (Cramer et al., 2014; Sowa et al., 2015; Armitage et al., 2011; Bae et al., 2018). The gold standard method to detect disruption of BBB is the visual inspection of post-contrast T1-images, but dynamic contrastenhanced MRI could quantify permeability of BBB, and the researchers obtained better specificity compared to other methods (Chen, Selvaganesan, Reich, Nair, \& Leigh, 2016). Despite multiple studies investigating cerebral blood perfusion parameters in multiple sclerosis by DSC-MRI, some recent investigation has shown that DCE-MRI can assess Cerebral Blood Flow (CBF) and Cerebral Blood Volume (CBV) by simulation. These measured parameters by DCE-MRI help detect vascular variations in NAWM and MS-related lesions (Ingrisch et al., 2017). These studies reported CBV reduced in MS lesions and WM of the patient compared to healthy volunteers. Also, CBF lacked a significant difference in the two groups (Ge et al., 2005; Ingrisch et al., 2012).

$\mathrm{CBV}$ and $\mathrm{CBF}$ were also obtained in $\mathrm{CE}$, and NCE lesions demonstrated an increase in $\mathrm{CBV}$ in $\mathrm{CE}$ lesions

Table 2. Permeability parameters in MS enhanced and non-enhanced lesions and NAWM

\begin{tabular}{cccc}
\hline Index & CE Lesions & NCE Lesions & NAWM \\
\hline Ktrans & $0.398(0.201-0.804)$ & $0.119(0.025-0.397)$ & $0.099(0.019-0.343)$ \\
Vb & $0.204(0.067-0.830)$ & $0.122(0.073-0.183)$ & $0.137(0.026-0.255)$ \\
Kep & $1.984(0.350-6.842)$ & $0.731(0.055-4.673)$ & $0.139(0.043-0.789)$ \\
\hline
\end{tabular}

MS: multiple sclerosis; CE: contrast-enhanced; NCE: non-contrast enhanced; RRMS: Relapsing-remitted multiple sclerosis; NAWM: normal-appearing white matter; CNS: central nervous system; BBB: blood-brain-barrier; MRI : magnetic resonance imaging; MS : multiple sclerosis; DCE: dynamic contrast-enhanced; SPGR: spoiled gradient recalled echo; AIF: Arterial input function; CBV: cerebral brain volume; CBF: cerebral brain flow; SPECT: single photon emission tomography; CA: contrast agent; DSC: dynamic susceptibility contrast; Gd: gadolinium ; ROI: region of interest; GM: gray matter; WM: white matter. 
Table.3. Comparison of the tracer kinetic parameters generated using the modified Tofts model between NEL and CEL

\begin{tabular}{ccccc}
\hline Parameters & Status & No. & Value(STD) & P \\
\hline \multirow{2}{*}{ Ktrans } & NEL & 13 & $0.17(0.10)$ & $<0.001$ \\
& CEL & 13 & $0.45(0.23)$ & 0.18 \\
Kep & NEL & 13 & $1.620(1)$ & 0.057 \\
Vb & CEL & 13 & $2.367(1.99)$ & $0.13(0.07)$ \\
\hline
\end{tabular}

The P-value is calculated using the Mann-Whitney U test.

NEUR:SCIENCE

MS: multiple sclerosis; CE: contrast-enhanced; NCE: non-contrast enhanced; RRMS: Relapsing-remitted multiple sclerosis; NAWM: normal-appearing white matter; CNS: central nervous system; BBB: blood-brain-barrier; MRI : magnetic resonance imaging; MS : multiple sclerosis; DCE: dynamic contrast-enhanced; SPGR: spoiled gradient recalled echo; AIF: Arterial input function; CBV: cerebral brain volume; CBF: cerebral brain flow; SPECT: single photon emission tomography; CA: contrast agent; DSC: dynamic susceptibility contrast; Gd: gadolinium ; ROI: region of interest; GM: gray matter; WM: white matter.

compared to NCE lesions. However, CBF has no significant difference between lesions and NAWM (Ge et al., 2005). However, recent studies have measured quantitative parameters like transfer rate constant between plasma and extravascular extracellular space to show subtle changes in BBB in MS patients. They showed an increase in measured parameters, which had significant differences between NAWM and WM lesions in MS brains (Cramer et al., 2014; Taheri et al., 2013). These variations are based on pathology changes in immunity and inflammatory cytokine and dysregulation of the
BBB in white matter lesions and white matter tissue (Ortiz et al., 2014; Varatharaj, \& Galea, 2017). Some studies investigated the relationship between measured perfusion parameters and EDSS. These studies confirmed our results and reported higher values in Ktrans and $\mathrm{Vb}$ in lesions compared with non-enhanced lesions and NAWM. However, they did not mention the relationship between these parameters and inflammatory activity of brain tissue during RRMS (Yin et al., 2018).

Table.4. Comparison of the tracer kinetic parameters generated using the modified-Tofts model between NEL, Brian NAWM and comparison between CEL, brain NAWM

\begin{tabular}{ccccc}
\hline Parameters & Status & No. & Value(STD) & P \\
\hline \multirow{3}{*}{ Ktrans } & NEL & 13 & $0.17(0.13)$ & 0.221 \\
& NAWM related to NEL brains & 13 & $0.12(0.08)$ & 0.001 \\
CEL & 13 & $0.45(0.23)$ & $0.11(0.08)$ & $<0.05$ \\
Kep & NAWM related to CEL brains & 13 & $1.620(1)$ & $<0.001$ \\
NEL & 13 & $0.252(0.19)$ & 0.152 \\
NAWM related to NEL brains & 13 & $2.367(1.99)$ & $0.175(0.02)$ \\
CEL & 13 & $0.13(0.07)$ & $0.15(0.07)$ \\
& NAWM related to CEL brains & 13 & $0.24(0.20)$ & $0.14(0.08)$ \\
\end{tabular}

The P-value is calculated using the Wilcoxon Ranked test.

MS: multiple sclerosis; CE: contrast-enhanced; NCE: non-contrast enhanced; RRMS: Relapsing-remitted multiple sclerosis; NAWM: normal-appearing white matter; CNS: central nervous system; BBB: blood-brain-barrier; MRI : magnetic resonance imaging; MS : multiple sclerosis; DCE: dynamic contrast-enhanced; SPGR: spoiled gradient recalled echo; AIF: Arterial input function; CBV: cerebral brain volume; CBF: cerebral brain flow; SPECT: single photon emission tomography; CA: contrast agent; DSC: dynamic susceptibility contrast; Gd: gadolinium ; ROI: region of interest; GM: gray matter; WM: white matter. 

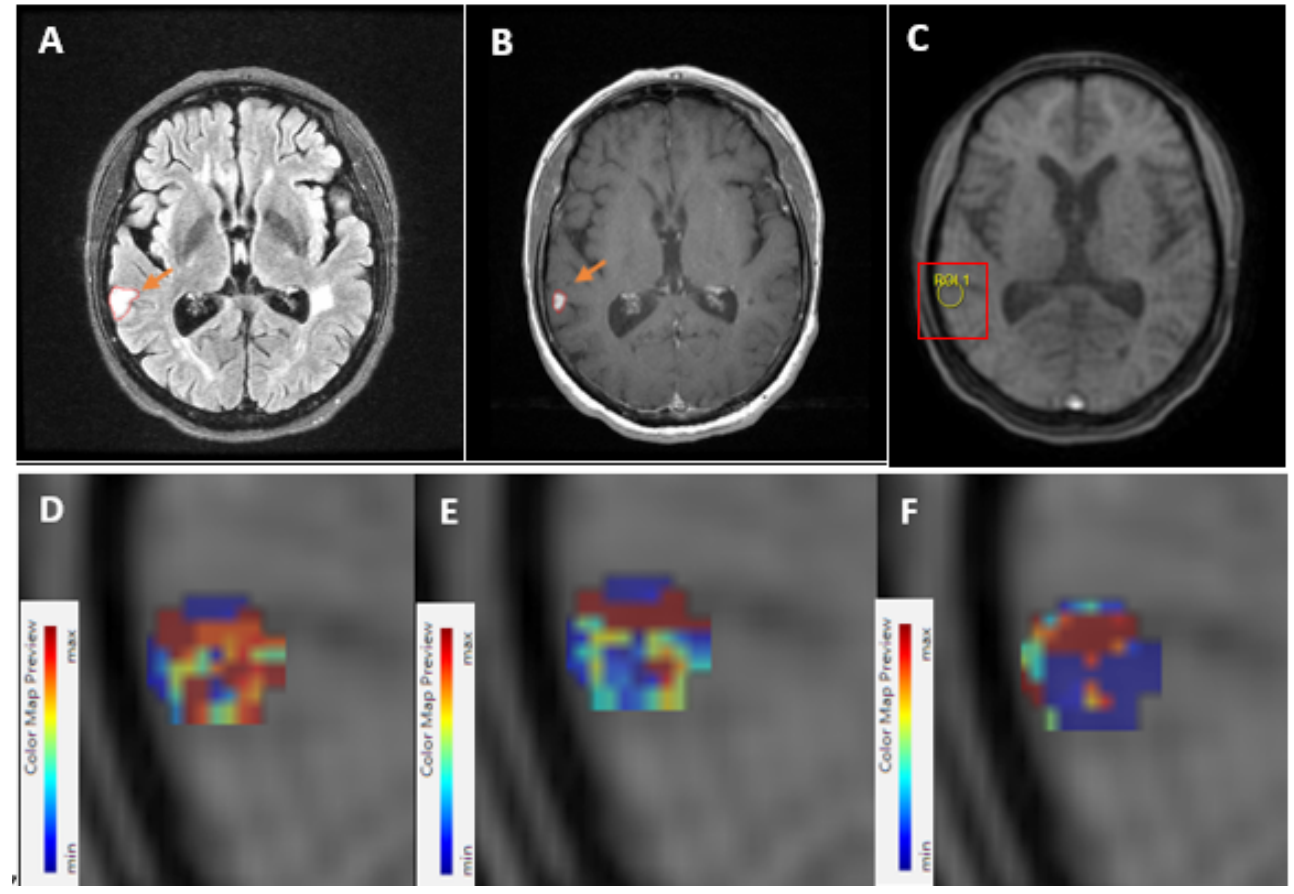

NEUR SCIENCE

Figure 1. Enhanced lesion in T2-FLAIR, Pre-contrast T1, and DCE-T1 in A, B, and C respectively

Also, Ktrans map and Vb map and Kep map in drawn square in DCE-T1in D, E and F. An orange sign in images locates the place of lesions.

Different models to analyze DCE-MRI images like Patlak were used to measure perfusion parameters of WM lesions and NAWM and increase in transfer rate in MS lesions compared to normal brain (Wiggermann et al., 2013). These models confirm our results in transfer rate constant of Ktrans, measured by the modified Toft model. However, other models like the Tikhonov twocompartment were used to quantify BBB leakage, and there were no significant differences between analyzing models (Cramer et al., 2014). Perfusion parameters were obtained by different analysis methods like Patlak in DCE-MRI in RRMS patients in this study (Xiong et al., 2019). Ktrans and other perfusion parameters like $\mathrm{Vb}$ have been introduced as appropriate biomarkers to show differences between NAWM and enhanced and non-enhanced lesions. Their results were similar to our results, too. Also, NAWM results show that Ktrans was significantly higher in periventricular NAWM and MS lesions which approved our measurements in lesions and NAWM of MS patients (Levesque et al., 2010). Many

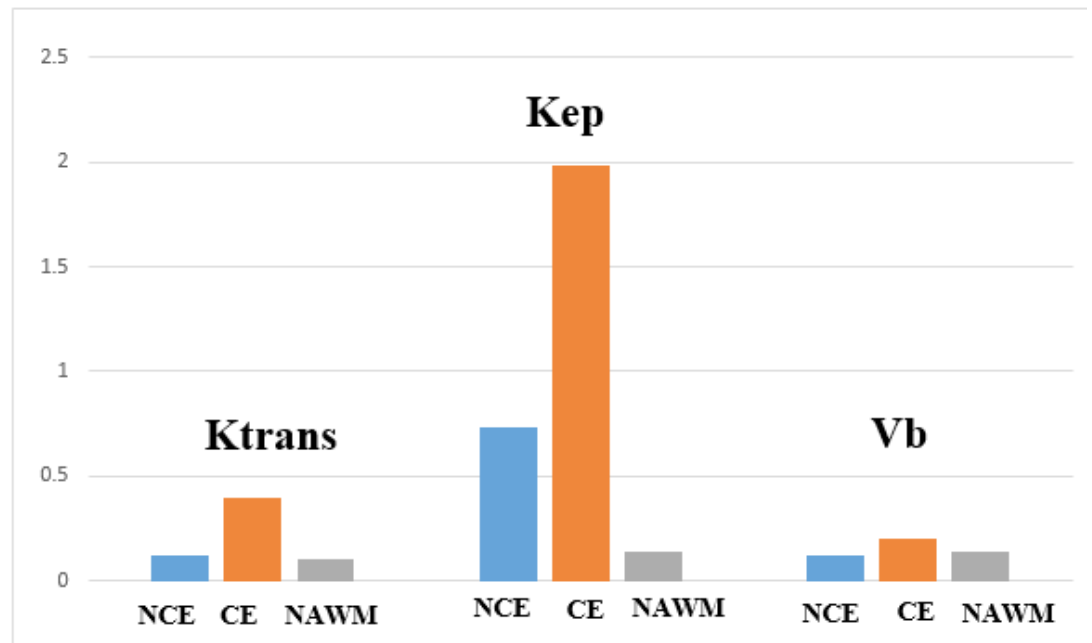

Figure.2. Bar plot of perfusion parameters of NCE, CE, and NAWM 


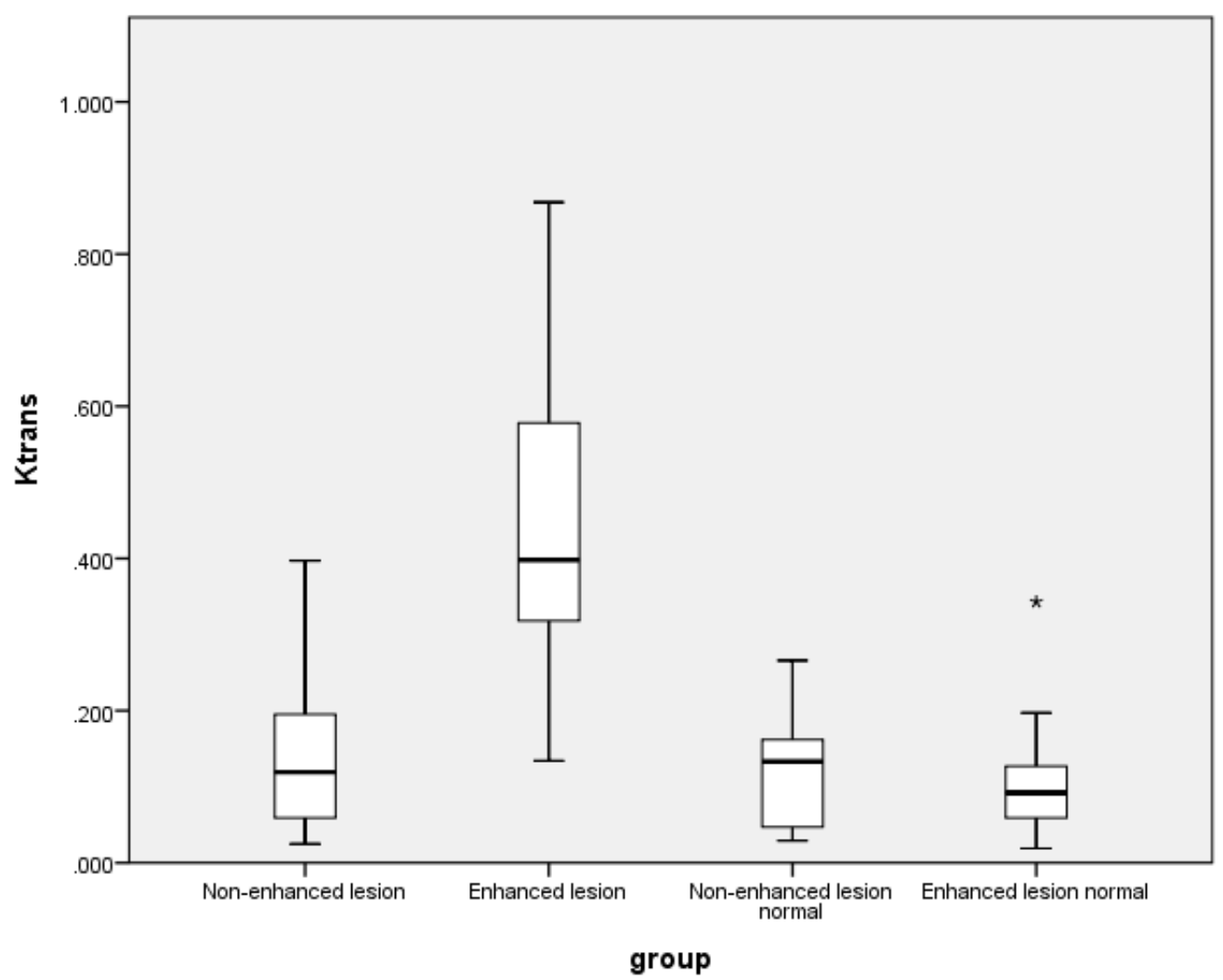

Figure 3. Box plot of Ktrans Value in enhanced and non-enhanced lesions and their NAWM

NEUR SCIENCE

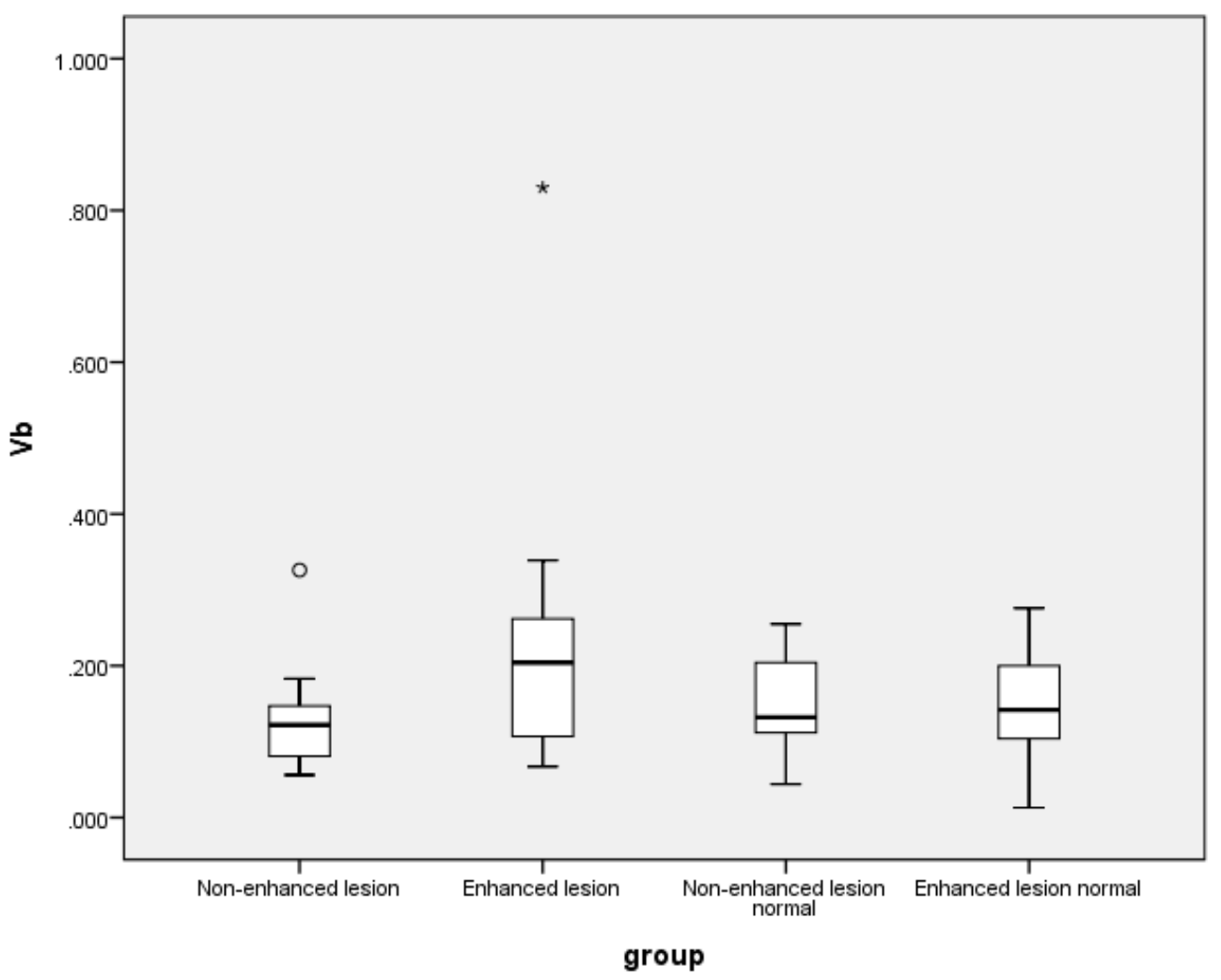

Figure.4. Box plot of $\mathrm{Vb}$ Value in enhanced and non-enhanced lesions and their NAWM 


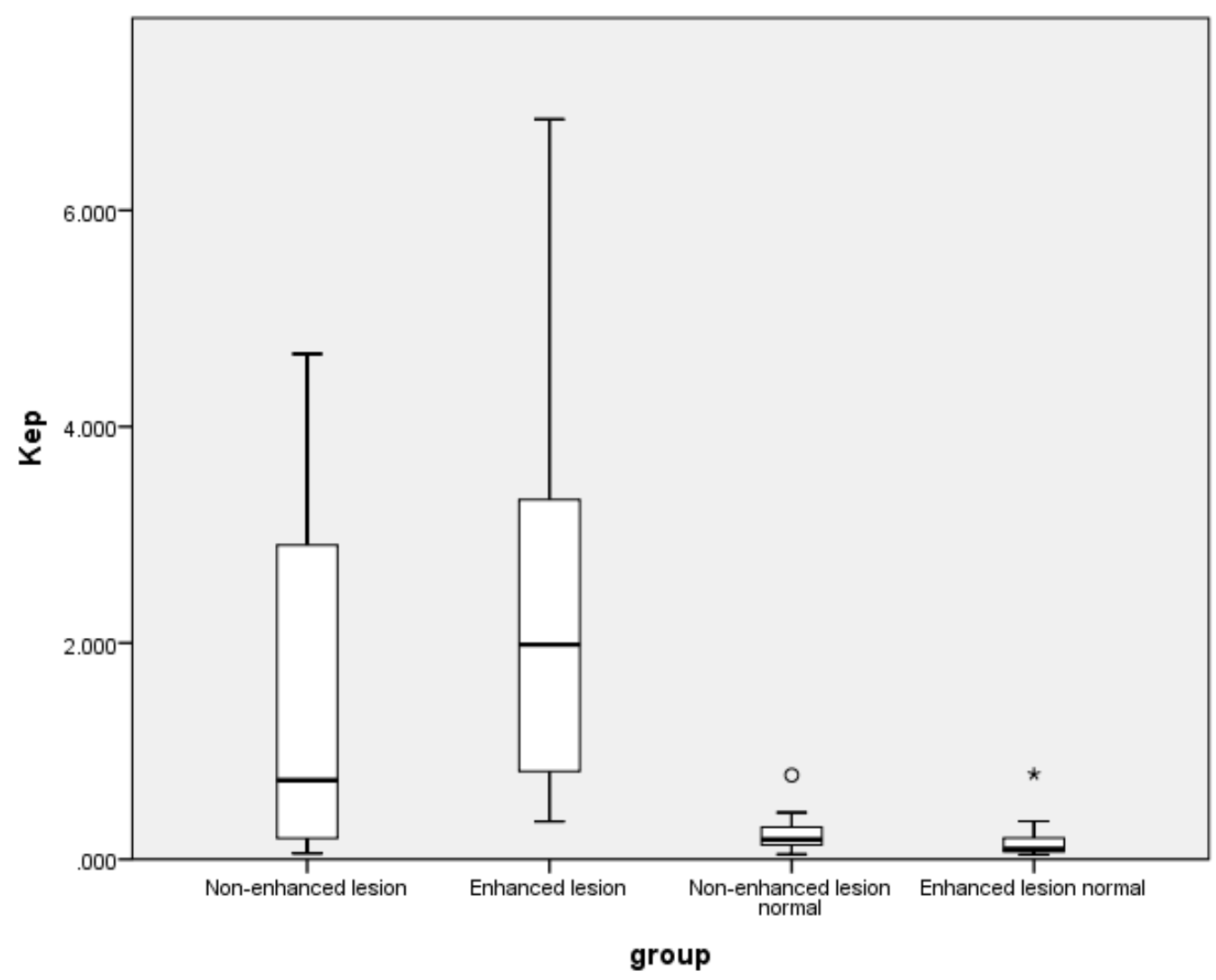

Figure.5. Box plot of Kep Value in enhanced and non-enhanced lesions and their NAWM

studies demonstrated that BBB functional impairment like high permeability could show inflammation and high brain immune cells activation associated with contrast enhancement in MR images. However, they mentioned that MR contrast uptake in MS Lesions could show a degree of inflammation (Quarantelli, 2015; Ortiz et al., 2014; Varatharaj., \& Galea, 2017).

Lacking information on the amount of BBB alterations in Gray Matter (GM) MS lesions has been investigated by many studies using many markers to characterize BBB structure in MS patients by plasma protein leakage and using perfusion imaging. These investigations revealed that $\mathrm{BBB}$ dysregulation is a common feature for white matter lesions but is not a critical change factor in forming gray matter MS lesions. Also, leakage of the BBB was measured using DCE-MRI by the value of Ktrans and compared with biological expectations. Their observation shows significant differences in gray matter and white matter, and the ratio of GM/WM cerebral blood flow was reported near-pathological results. DCEMRI technique revealed faster leakage in GM compared with WM in healthy controls and suggested that perfusion has a greater value in GM that corresponds to higher vascular surface area per unit tissue volume, so it has an important role in the measurement of Ktrans (Thrippleton, 2019; van Horssen, Brink, de Vries, van der Valk, \& Bø, 2007; Varatharaj, Liljeroth, M., Darekar, Larsson, Galea, \& Cramer, 2019).

Despite the difference in the analysis method of our research and the mentioned study, the same results were obtained. So our result approved the approach, i.e., high permeability in lesions that uptake contrast agents. So, these parameters (Ktrans, Kep, Vb) could be good indexes of BBB impairment and inflammation in MS (Varatharaj et al., 2019). Other studies mentioned that DCE-MRI gives us quantitative information about the function of the vascular system in brain tumors. However, they confirmed that Ktrans is the best parameter to detect the state of BBB integrity and pointed out that perfusion parameters like Ktrans could provide information to the presence of inflammation in MS-related Lesions. They concluded that DCE-MRI appears to be helpful in the evaluation of normal-appearing white matter and therapeutic response assessment in MS (Gupta, 2018).

\section{Conclusion}

The study's preliminary data can distinguish between acute and chronic lesions, detect MS-enhanced plaques, 
and be considered a hallmark of inflammatory activity in MS active lesions. We have used the DCE-MRI method to determine perfusion parameters to show BBB impairment in active lesions compared to chronic lesions in RRMS patients. Our results have shown that high inflammation interaction can change perfusion MRI parameters like Ktrans, Kep, and Vb in lesions that uptake Gd-contrast agents. Study measurements demonstrated an increased value in contrast-enhancing lesions, indicating dysfunction in the permeability of $\mathrm{BBB}$, which happened during high immune cell interactions in brain tissue during inflammation. So, Ktrans could be a good index to show BBB dysregulation in white matter lesions. These parameters can confirm the activation of inflammatory agents in enhanced lesions. Thus, we could determine a combination of quantitative modalities in MRI to detect neuroinflammation and use these modalities to highlight inflammation in other brain disorders.

\section{Study limitations}

This study has some limitations. These limitations include a small sample size and the absence of healthy people in the study. Despite less invasion of DCE-MRI compared with PET (Positron Emission Tomography) and SPECT studies, the obtained images with Gd-CA in healthy people are controversial among many researchers. So our Ethics Committee disapproved of using the CA on normal people. As a result, our limitation in obtaining images by Arterial Spin Labeling (ASL) perfusion imaging in MRI scanners without using CA is one of the major reasons not to include normal people. So our suggestion for future studies is to use the ASL method to investigate better the differences between NAWM in MS patients and normal WM in healthy individuals.

Our consultant neurologist mentioned that MS drugs could affect our results, so we obtained our images in the longest time between taking the drug. However, future research studies could investigate patients knowing the effect of a certain drug on BBB. Our software provided modified and standard Toft models, and a previous study showed modified Toft had acceptable results (Cramer et al., 2014). However, this issue will need more investigation for future works. To measure perfusion parameters, semi-manually ROIs were located in images that subjectively depended on the radiologist. Also, the sample size could affect the results. So, we advised a study with a larger sample size. In summary, this study provided measurements of perfusion parameters and suggested a method to show BBB impairment in MS brains.

\section{Ethical Considerations}

\section{Compliance with ethical guidelines}

The study was approved by Tehran University of Medical Sciences (Code: IR.TUMS.MEDICINE. REC.1396.2662).

\section{Funding}

This study was funded by the Tehran University of Medical Sciences (95-04-30-33430).

\section{Authors' contributions}

All authors equally contributed to preparing this article.

\section{Conflict of interest}

The authors declared no conflict of interest.

\section{Acknowledgments}

The authors thank the MS Clinic of Imam Hospital, Tehran, Iran, to introduce the proper patients and Imam Khomeini Medical Imaging Center, Tehran, Iran, for imaging data acquisition. Prof.Oghabian as corresponding author and supervisor of Asieh Fatemidokht $\mathrm{PhD}$ thesis. This article was a part of Asieh Fatemidokht $\mathrm{PhD}$ thesis and she was first author. Prof. Harrichian was a major consultant and reviewer of artricle.

\section{References}

Absinta, M., Sati, P., \& Reich, D. S. (2016). Advanced MRI and staging of multiple sclerosis lesions. Nature Reviews Neurology, 12(6), 358-68. [DOI:10.1038/nrneurol.2016.59] [PMID] [PMCID]

Armitage, P. A., Farrall, A. J., Carpenter, T. K., Doubal, F. N \& Wardlaw, J. M. (2011). Use of dynamic contrast-enhanced MRI to measure subtle blood-brain barrier abnormalities. Magnetic Resonance Imaging, 29(3), 305-14. [DOI:10.1016/j. mri.2010.09.002] [PMID] [PMCID]

Bae, J., Zhang, J., Wadghiri, Y. Z., Minhas, A. S., Poptani, H., Ge, Y., et al. (2018). Measurement of blood-brain barrier permeability using dynamic contrast-enhanced magnetic resonance imaging with reduced scan time. Magnetic Resonance in Medicine 80(4), 1686-96. [DOI:10.1002/mrm.27145] [PMID] [PMCID]

Barkhof, F. (2002). The clinico-radiological paradox in multiple sclerosis revisited. Current Opinion in Neurology, 15(3), 23945. [DOI:10.1097/00019052-200206000-00003] [PMID]

Chen, N., Selvaganesan, K., Reich, D., Nair, G., \& Leigh, R. (2016). A comparison of two methods for quantifying bloodbrain barrier disruption, with application to multiple sclerosis 
(S45. 006). Neurology, 86(16 Supplement). https://n.neurology.org/content/86/16_Supplement/S45.006.short

Cramer, S. P., \& Larsson, H. B. (2014). Accurate determination of blood-brain barrier permeability using dynamic contrastenhanced T1-weighted MRI: A simulation and in vivo study on healthy subjects and multiple sclerosis patients. Journal of Cerebral Blood Flow and Metabolism, 34(10), 1655-65. [DOI:10.1038/jcbfm.2014.126] [PMID] [PMCID]

Cramer, S. P., Simonsen, H., Frederiksen, J. L., Rostrup, E., \& Larsson, H. B. (2014). Abnormal blood-brain barrier permeability in normal appearing white matter in multiple sclerosis investigated by MRI. NeuroImage: Clinical, 4, 182-9. [DOI:10.1016/j.nicl.2013.12.001] [PMID] [PMCID]

Eftekhari, E., Hojjat, S. P., Vitorino, R., Carroll, T. J., Cantrell, C. G., Lee, L., et al. (2017). Normal appearing white matter permeability: A marker of inflammation and information processing speed deficit among relapsing remitting multiple sclerosis patients. Neuroradiology, 59(8), 771-80. [DOI:10.1007/ s00234-017-1862-7] [PMID]

Filippi, M., \& Rocca, M. A. (2011). MR imaging of multiple sclerosis. Radiology, 259(3), 659-81. [DOI:10.1148/radiol.11101362] [PMID]

Gaitan, M. I., Shea, C. D., Evangelou, I. E., Stone, R. D., Fenton, K M., Bielekova, B., et al. (2011). Evolution of the blood-brain barrier in newly forming multiple sclerosis lesions. Annals of Neurology, 70(1), 22-9. [DOI:10.1002/ana.22472] [PMID] [PMCID]

Ge, Y., Law, M., Johnson, G., Herbert, J., Babb, J. S., Mannon, L. J., et al. (2005). Dynamic susceptibility contrast perfusion MR imaging of multiple sclerosis lesions: Characterizing hemodynamic impairment and inflammatory activity. American Journal of Neuroradiology, 26(6), 1539-47. [PMID] [PMCID]

Gilad, R., Lampl, Y., Eilam, A., Boaz, M., \& Loyberboim, M. (2012). SPECT-DTPA as a tool for evaluating the blood-brain barrier in post-stroke seizures. Journal of Neurology, 259(10), 2041-4. [DOI:10.1007/s00415-012-6445-2] [PMID]

Gupta, R. K. (2018). Quantification of blood brain barrier using DCE MRI in multiple sclerosis-Technical issues and its possible role in routine clinical practice. Neurology India, 66(3), 671-2. [DOI:10.4103/0028-3886.232323] [PMID]

Hagens, M., van Berckel, B., \& Barkhof, F. (2016). Novel MRI and PET markers of neuroinflammation in multiple sclerosis. Current Opinion in Neurology, 29(3), 229-36. [DOI:10.1097/ WCO.0000000000000326] [PMID]

Ingrisch, M., Sourbron, S., Herberich, S., Schneider, M. J., Kümpfel, T., Hohlfeld, R., et al. (2017). Dynamic contrastenhanced magnetic resonance imaging suggests normal perfusion in normal-appearing white matter in multiple sclerosis. Investigative Radiology, 52(3), 135-41. [DOI:10.1097/ RLI.0000000000000320] [PMID]

Ingrisch, M., Sourbron, S., Morhard, D., Ertl-Wagner, B., Kumpfel, T., Hohlfeld, R., et al. (2012). Quantification of perfusion and permeability in multiple sclerosis: Dynamic contrast-enhanced MRI in 3D at 3T. Investigative Radiology, 47(4), 252-8. [DOI:10.1097/RLI.0b013e31823bfc97] [PMID]

Jurcoane, A., Wagner, M., Schmidt, C., Mayer, C., Gracien, R. M., Hirschmann, M., et al. (2013). Within-lesion differences in quantitative MRI parameters predict contrast enhancement in multiple sclerosis. Journal of Magnetic Resonance Imaging, 38(6), 1454-61. [DOI:10.1002/jmri.24107] [PMID]
Levesque, I. R., Giacomini, P. S., Narayanan, S., Ribeiro, L. T. Sled, J. G., Arnold, D. L., et al. (2010). Quantitative magnetization transfer and myelin water imaging of the evolution of acute multiple sclerosis lesions. Magnetic Resonance in Medicine, 63(3), 633-40. [DOI:10.1002/mrm.22244] [PMID]

Li, W., Long, J. A., Watts, L. T., Jiang, Z., Shen, Q., Li, Y., et al. (2014). A quantitative MRI method for imaging blood-brain barrier leakage in experimental traumatic brain injury. PLoS One, 9(12), e114173. [DOI:10.1371/journal.pone.0114173] [PMID] [PMCID]

Nagaraja, T. N., Nagesh, V., Ewing, J. R., Whitton, P. A., Fenstermacher, J. D., \& Knight, R. A. (2007). Step-down infusions of Gd-DTPA yield greater contrast-enhanced magnetic resonance images of BBB damage in acute stroke than bolus injections. Magnetic Resonance Imaging, 25(3), 311-8. [DOI:10.1016/j.mri.2006.09.003] [PMID] [PMCID]

Ortiz, G. G., Pacheco-Moises, F. P., Macias-Islas, M. A., FloresAlvarado, L. J., Mireles-Ramirez, M. A., Gonzalez-Renovato, E. D., et al. (2014). Role of the blood-brain barrier in multiple sclerosis. Archives of Medical Research, 45(8), 687-97. [DOI:10.1016/j.arcmed.2014.11.013] [PMID]

Popescu, B. F. G., Pirko, I., \& Lucchinetti, C. F. (2013). Pathology of multiple sclerosis: Where do we stand? Continuum: Lifelong Learning in Neurology, 19(4 Multiple Sclerosis), 901-21. [DOI:10.1212/01.CON.0000433291.23091.65] [PMID] [PMCID]

Quarantelli, M. (2015). MRI/MRS in neuroinflammation: Methodology and applications. Clinical and Translational Imaging, 3(6), 475-89. [DOI:10.1007/s40336-015-0142-y] [PMID] [PMCID]

Roberts, H. C., Roberts, T. P., Brasch, R. C., \& Dillon, W. P. (2000). Quantitative measurement of microvascular permeability in human brain tumors achieved using dynamic contrast-enhanced MR imaging: Correlation with histologic grade. American Journal of Neuroradiology, 21(5), 891-9. [PMID] [PMCID]

Schabel, M. C. (2012). A unified impulse response model for DCE-MRI. Magnetic Resonance in Medicine, 68(5), 1632-46. [DOI:10.1002/mrm.24162] [PMID]

Sowa, P., Bjornerud, A., Nygaard, G. O., Damangir, S., Spulber, G., Celius, E. G., et al. (2015). Reduced perfusion in white matter lesions in multiple sclerosis. European Journal of Radiology, 84(12), 2605-12. [DOI:10.1016/j.ejrad.2015.09.007] [PMID]

Taheri, S., Rosenberg, G. A., \& Ford, C. (2013). Quantification of blood-to-brain transfer rate in multiple sclerosis. Multiple Sclerosis and Related Disorders, 2(2), 124-32. [DOI:10.1016/j. msard.2012.09.003] [PMID] [PMCID]

Thompson, A. J., Banwell, B. L., Barkhof, F., Carroll, W. M., Coetzee, T., Comi, G., et al. (2018). Diagnosis of multiple sclerosis: 2017 revisions of the McDonald criteria. The Lancet. Neurology, 17(2), 162-73. [DOI:10.1016/S1474-4422(17)304702] [PMID]

Thrippleton, M. J. (2019). MRI measurement of blood-brain barrier leakage: Filling the gaps. The Journal of Physiology, 597(3), 667-8. [DOI:10.1113/JP277425] [PMID] [PMCID]

Tofts, P. S., Brix, G., Buckley, D. L., Evelhoch, J. L., Henderson, E., Knopp, M. V., et al. (1999). Estimating kinetic parameters from dynamic contrast-enhanced T(1)-weighted MRI of a diffusable tracer: Standardized quantities and symbols. Journal 
of Magnetic Resonance Imaging, 10(3), 223-32. [DOI:10.1002/ (SICI)1522-2586(199909)10:33.0.CO;2-S] [PMID]

Tourdias, T., \& Dousset, V. (2013). Neuroinflammatory imaging biomarkers: Relevance to multiple sclerosis and its therapy. Neurotherapeutics, 10(1), 111-23. [DOI:10.1007/s13311-0120155-4] [PMID] [PMCID]

Varatharaj, A., \& Galea, I. (2017). The blood-brain barrier in systemic inflammation. Brain, Behavior, and Immunity, 60, 1-12. [DOI:10.1016/j.bbi.2016.03.010] [PMID]

Varatharaj, A., Liljeroth, M., Darekar, A., Larsson, H. B. W., Galea, I., \& Cramer, S. P. (2019). Blood-brain barrier permeability measured using dynamic contrast-enhanced magnetic resonance imaging: A validation study. Journal of Physiology, 597(3), 699-709. [DOI:10.1113/JP276887] [PMID] [PMCID]

van Horssen, J., Brink, B. P., de Vries, H. E., van der Valk, P., \& Bø, L. (2007). The blood-brain barrier in cortical multiple sclerosis lesions. Journal of Neuropathology \& Experimental Neurology, 66(4), 321-8. [DOI:10.1097/nen.0b013e318040b2de] [PMID]

Wiggermann, V., Hernandez Torres, E., Vavasour, I. M., Moore, G. R., Laule, C., MacKay, A. L., et al. (2013). Magnetic resonance frequency shifts during acute MS lesion formation. Neurology, 81(3), 211-8. [DOI:10.1212/WNL.0b013e31829bfd63] [PMID] [PMCID]

Xiong, H., Yin, P., Li, X., Yang, C., Zhang, D., Huang, X., et al (2019). The features of cerebral permeability and perfusion detected by dynamic contrast-enhanced magnetic resonance imaging with Patlak model in relapsing-remittitng multiple sclerosis. Therapeutics and Clinical Risk Management, 15, 233-40. [DOI:10.2147/TCRM.S189598] [PMID] [PMCID]

Yin, P., Xiong, H., Liu, Y., Sah, S. K., Zeng, C., Wang, J., et al. (2018). Measurement of the permeability, perfusion, and histogram characteristics in relapsing-remitting multiple sclerosis using dynamic contrast-enhanced MRI with extended Tofts linear model. Neurology India, 66(3), 709-15. [DOI:10.4103/0028-3886.232324] [PMID] 